Original Article

\title{
STATUS OF LIPID PEROXIDATION AND TOTAL ANTIOXIDANT CAPACITY IN REGULAR VOLUNTARY BLOOD DONORS
}

\author{
Chandrika ${ }^{1}$, Suchetha Kumari ${ }^{1}$, Jayaprakash Shetty ${ }^{2}$, Kathyayani ${ }^{2}$, Sharada ${ }^{3}$ \\ ${ }^{1}$ Department of Pathology, ${ }^{1}$ Department of Biochemistry and ${ }^{2}$ Department of Community Medicine, \\ K.S. Hegde Medical Academy, Deralakatte, Mangalore - 575 018, Karnataka, India

\section{Correspondence :} \\ Chandrika \\ Blood Bank Officer, Department of Pathology, K.S. Hegde Medical Academy, \\ Deralakatte, Mangalore - 575 018, Karnataka, India. \\ E-mail : chandrika_valal@yahoo.com
}

\begin{abstract}
:
Oxygen free radicals and products of oxidative cell damage are known as biomarkers that escalate in the pathophysiology of many diseases. The aim of this study is to evaluate association between blood donation and total antioxidant capacity and lipid peroxidation product malondialdehyde(MDA). The levels of MDA and total antioxidant capacity(TAC) were studied in serum of 90 male voluntary blood donors aged from 20 to 45 years attending K. S. Hegde charitable hospital, Blood bank, for determination of lipid peroxidation and antioxidant status, respectively. The donors were divided into nine groups depending on number of blood donation. With increase in number of blood donation the serum MDA level were significantly reduced $(p<0.01)$ but total antioxidant activity was significantly increased $(p<0.01)$. High frequency of blood donors had evidence of decreased lipid peroxidation and enhanced activity of total antioxidants when compared with low frequency blood donors. which can be considered as one of the fringe benefits of regular blood donation.
\end{abstract}

Keywords : Blood donation, lipid peroxidation, malondiadehyde, total antioxidant capacity

\section{Introduction :}

Free radicals are highly reactive molecules generated by biochemical redox reactions which occur as a part of normal cell metabolism and in the course of free radical mediated diseases such as cancer, diabetes mellitus cardiovascular and renal diseases. ${ }^{1}$ Oxidative stress represents an imbalance between the production and manifestation of reactive oxygen species and a biological system's ability to readily detoxify the reactive intermediates or to repair the resulting damage. Free radicals may cause lipid peroxidation, the level of lipid peroxidation expressed as malondialdehyde, that damage all components of the cell, including proteins, lipids, and DNA. Malondiadehyde is a metabolic product of peroxidative reactions (auto-oxidative) of lipids exposed to oxygen. ${ }^{2}$ It serves as a reliable marker of lipid peroxidation. ${ }^{3}$ Free radicals are eliminated from the body by their interaction with non enzymic antioxidants such as uric acid, albumin, bilirubin, vitamin $A, C, E$, glutathione, peroxidase, superoxide dismutase and catalase. ${ }^{1,4}$

Blood is permanently exposed to oxidative stress and therefore it has high antioxidant capacity. ${ }^{1,5}$ Significantly decreased antioxidants and markedly increased MDA can cause metabolic disorders, increase oxidative damage and oxidative stress in human body.

The present study aims to determine the alteration of serum lipid peroxidation (by measuring the level of MDA) and total antioxidant capacity in regular blood donors.

\section{Materials and methods :}

The reference population consisted of 90 male volunteer blood donors aged from 25 to 50 years attending K.S. Hegde charitable hospital, Blood Bank. The ethical committee of our institution approved the trial. We obtained informed consent from all participants.

The inclusion criteria included no history of minor surgery during the previous 72 hours, no major surgery within 6 months, no current infectious or non infectious disease, notably cardiovascular disease, diabetes, polycyaethemia vera, no addiction to drugs and/or alcohol, no history of tattoo in the previous 6 months, minimum weight of $50 \mathrm{kgs}$, systolic and diastolic blood pressure between 
100-120mmHg and $60-.80 \mathrm{mmHg}$, respectively. Subjects were divided into nine groups depending on number of blood donations; i.e 0, 3, 4, 5, 6, 7, 8, 9, 10 and 10 persons in each group, respectively. Questionnaire was prepared for data collection.

Just before blood donation $2 \mathrm{ml}$ of venous blood was taken. Serum was separated by centrifugation. The serum was recovered and transferred to fresh tube for determination of MDA level and total antioxidant capacity(TAC).

\section{Determination of MDA level :}

Lipid peroxidation was estimated by measurement of thiobarbutric acid by the method of Buege and Aust(1978).Serum-100 $\mu \mathrm{L}$ was diluted to $500 \mu \mathrm{L}$ with distilled water. To the diluted sample $1 \mathrm{~mL}$ of TCA-TBA- $\mathrm{HCl}$ (Trichloroacetic acid- thiobarbutric acid-hydrochloric acid) reagent was added. The samples were kept in boiling water bath for 15 minutes. The reaction mixture was cooled and centrifuged. The supernatant was taken and the optical density of the pink colour formed was read at $535 \mathrm{~nm}$. A blank was also maintained simultaneously by taking $500 \mu \mathrm{L}$ of water instead of sample in the reaction mixture. The concentration of malondialdehyde in the sample was determined by plotting the obtained absorbance against the standard graph. The optical density of the pink colour formed is directly proportional to the concentration of malondialdehyde in the given sample.

\section{Enzymatic determination of total antioxidant capacity :}

Total antioxidant capacity was determined by the method of Prieto et.al.,1999.Exactly $100 \mu \mathrm{L}$ of serum sample was pipetted out into a clean test tube and $100 \mu \mathrm{L}$ of $5 \% \mathrm{TCA}$ (Trichloroacetic acid) was added to it to precipitate out the proteins in the sample, the mixture was then allowed to stand for about five minutes and centrifuged. $100 \mu \mathrm{L}$ of the clear supernatant was transferred into a clean test tube and $1 \mathrm{~mL}$ of total antioxidant capacity( TAC) reagent was added to it and the mixture was then incubated in water bath at $90^{\circ} \mathrm{C}$ for 90 minutes. A blank was also maintained simultaneously by substituting $100 \mu \mathrm{L}$ of water instead of sample in the reaction mixture. Following the incubation the reaction mixture was cooled and the optical density of the greenish to bluish colour formed was read at $695 \mathrm{~nm}$ against blank. The total antioxidant capacity in the sample is obtained by plotting the obtained absorbance against the standard graph.

\section{Data analysis :}

Results were expressed as mean \pm standard error of mean (SEM). Correlations were evaluated using Pearson's correlation coefficient. The data were subjected to one way ANOVA. The predetermined upper limit of significance throughout this investigation was $p<0.05$. all analyses were done using SPSS 11.5.

\section{Results :}

In present study we determined the serum level of malondialdejyde and total antioxidant status. Mean and standard error of mean for each group was calculated (Table I). There is a significant decrease in serum malondialdehyde level (Graph I)and significant increase in total antioxidant capacity (Graph II) with increase in number of blood donation $(p<0.01)$. MDA showed significant correlation with total antioxidant capacity ( $r=-$ 0.880, p <0.001 ) in group II with blood donors having donated three times. (Table I) (Diagram I)

\section{Discussion :}

The current findings demonstrate evidence of increase activity of antioxidants, decrease oxidative stress and decrease lipid peroxidation in high frequency blood donors. Lipid peroxidation is a free radical generating process which occurs on every membranous structure of the cell. ${ }^{2}$ Free radicals are known to be involved in pathologies including atherosclerosis, cancer and hypertension. ${ }^{7}$ Our finding of decreased MDA in high frequency donors is consistent with the hypothesis that high frequency blood donation is associated with decrease oxidative stress. To best of our knowledge, only one study, has been conducted on blood donors to find association between blood donation frequency, antioxidant enzymes and lipid peroxidation, results suggested evidence of reduction of body iron stores, reduced oxidative stress and enhanced activity of antioxidant enzymes like superoxide dismutase, in 
voluntary blood donors. ${ }^{2}$ Few studies have examined the association of plasma iron levels and plasma MDA in human population. Results from study in patients with coronary artery disease, showed an association of high serum ferritin with increase lipid peroxidation. ${ }^{8}$ Number of studies were conducted to know the association between repeated blood donation and iron status., ${ }^{9,10,11}$

To our knowledge, this is the second study to determine association between blood donation, lipid peroxidation and antioxidant capacity in regular blood donors.

\section{Conclusion :}

Increase in number of blood donation was associated with evidence of reduced oxidative stress and enhanced activity of antioxidants in regular voluntary blood donors, which can reduce the risk of certain diseases. Additional studies are required to evaluate the effect of blood donation on lipid peroxidation and antioxidant status in larger population by dividing groups according to the frequency of blood donation per year in different age groups.

Table I. Serum malondialdehyde and total antioxidant capacity in subjects based on number of blood donation and its correlation

\begin{tabular}{|l|l|l|l|l|}
\hline Groups $(\mathrm{n}=10)$ & $\begin{array}{c}\text { MDA } \\
\text { Mean } \pm \text { SEM }\end{array}$ & $\begin{array}{l}\text { TAC } \\
\text { Mean } \pm \text { SEM }\end{array}$ & $\begin{array}{l}\text { Pearson's } \\
\text { correlation ' } r\end{array}$ & p value \\
\hline I (0 time) & $2.68 \pm 0.079$ & $183.74 \pm 1.94$ & 0.229 & 0.525 \\
\hline II (3 times) & $2.15 \pm 0.07$ & $185.92 \pm 2.64$ & $-0.880 * *$ & $0.001 * *$ \\
\hline III (4 times) & $2.16 \pm 0.07$ & $191.44 \pm 1.05$ & -0.401 & 0.251 \\
\hline IV (5 times) & $2.45 \pm 0.06$ & $203.40 \pm 1.35$ & 0.269 & 0.452 \\
\hline V ( 6 times) & $1.90 \pm 0.10$ & $226.56 \pm 3.14$ & -0.277 & 0.438 \\
\hline VI (7 times) & $2.24 \pm 0.10$ & $224.85 \pm 3.78$ & 0.592 & 0.071 \\
\hline VII (8 times) & $2.08 \pm 0.06$ & $237.73 \pm 4.03$ & -0.611 & 0.061 \\
\hline VIII ( 9 times) & $2.08 \pm 0.05$ & $235.33 \pm 5.50$ & -0.144 & 0.692 \\
\hline IX (10 times) & $2.08 \pm 0.11$ & $270.40 \pm 12.47$ & -0.154 & 0.672 \\
\hline
\end{tabular}

Abbreviations: MDA, malondialdehyde; TAC, total antioxidant capacity

** Correlation is significant at 0.01 level ( 2 tailed)

Diagram 1. Scatter plot of serum malondialdehyde and total antioxidant capacity

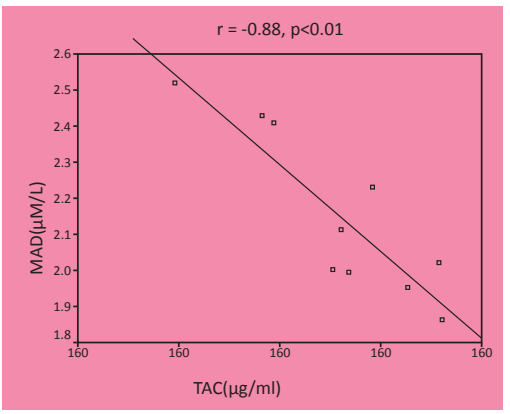

Abbreviations : MDA, malondialdehyde; TAC total antioxidant capacity

Graph I. Error bar graph for serum malondialdehyde (MDA) with mean and standard deviation

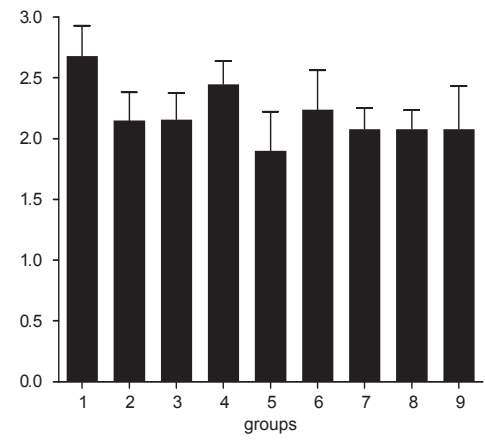

Graph II. Error bar graph for total antioxidant capacity (TAC) with mean and standard deviation

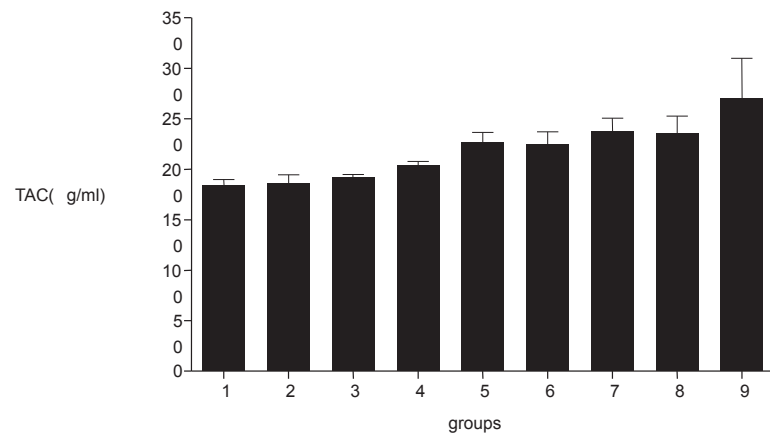




\section{References :}

1. Abdoljalal M.Alterations in plasma lipid peroxidation and total antioxidant status during storage of blood. Pak J Biol Sciences 2006;9(13):2520-23.

2. Mehrabani M, Djalali M, Sadeghi MR, Hajibeigi B, Zeraati H, Fatehi F et al. Association between blood donation frequency, antioxidant enzymes and lipid peroxidation. Acta Medica Iranica.2008;46(5):36165

3. Boaz M, Matas Z, Biro et al. Comparison of hemostatic factors and serum malondialdehyde as predictive factors of cardiovascular disease in hemodialysis patients. Am J Kidney Dis;34:438-44.

4. Kohen R, Chevion S, Schartz R, Berry EM. Evaluation of the total low molecular weight antioxidant activity of plasma in health and disease:A new approach. Cell Pharmacol. 1996;3:355-59.

5. Lewin G, Popov I. The antioxidant system of organism. Theoretical basis and practical consequences. Med Hypotheses.1994;42:269-75.

6. Zweier JL, Broderick R, Kuppuswamy P, Thompson GS, Lutty GA Determination of the mechanism of free radical generation in human aortic endothelial cells exposed to anoxia and reoxygenation. J Biol Chem. 1994;269(39):24156-62.
7. Ammala C, Eliasson L, Bokvist K, Larsson O, Ashcroft FM, Rorsman P. Exocytosis elicited by action potentials and voltage-clamp calcium currents in individual mouse pancreatic B-cells. JPhysiol. 1993;472:665-88.

8. Yesilbursa D, Serdar Z, Serdar A, Dirican M, Gemici K, Ozdemir A, Turel $B$, Cordan J. The relationship of serum ferritin with malondialdehyde concentration in patients with coronary artery disease:Ferritin and oxidative stress in CAD. International Journal of Angiology.2001;10(2):88-91.

9. Romila M, Neelam M, Sabita B, Harsh M, Ravikumar A. Evaluation of iron stores in blood donors by serum ferritin. Indian J Med Res 2006;124:641-46.

10. S B Kwa. The effect of repeated blood donation on the haemoglobin, haematocrit and serum iron values of regular blood donors. Singapore medical journal 1969;10(3):139-47.

11. Abdullah SM. The effect of repeated blood donations on the iron status of male Saudi blood donors. Blood Transfus 2011;9(2):67-71. 